\title{
Age-dependent physiological changes, medicines and sex-influenced types of falls
}

\author{
Aging, medicines and falls
}

Snezana Kusljic, Sachin Perera \& Elizabeth Manias

\section{Snezana Kusljic}

Department of Nursing, The University of Melbourne, Melbourne, Australia; and The Florey Institute of Neuroscience and Mental Health, Melbourne, Australia

\section{Sachin Perera}

Department of Medicine, The Royal Melbourne Hospital, The University of Melbourne, Melbourne, Australia

Elizabeth Manias

Department of Nursing and Department of Medicine, The Royal Melbourne Hospital, The University of Melbourne, Melbourne, Australia; and School of Nursing and Midwifery, Deakin University, Melbourne, Australia 


\section{ABSTRACT}

Background/Study context: We investigated various parameters related to falls including agedependent physiological changes, regular medicine use and different types of falls experienced. There is a lack of research investigating the impact of health status, sex, polypharmacy and ageing on different types of falls such as unspecified fall on the same level, mechanical fall on the same level relating to slipping, tripping or loosing balance, fall from a chair, vehicle and fall as a result of syncope, fall from steps or stairs, and fall from the height.

Methods: The study included a random sample of 250 older patients, which comprised $10 \%$ of the total number of patients $(n=2,492)$, admitted to a large scale academic hospital following a fall. Patients' medicine and illness history, types of falls, liver, renal, and sensory function were collected. Univariate analysis was used to examine associations between the type of fall and explanatory variables, followed by multinominal logistic regression analysis.

Results: There was a significant association between the type of fall and sex, $\mathrm{p}=0.01$, and between the type of fall and regular medicine use, $p=0.002$. The multinominal logistic regression analysis revealed that the full model, which considered all explanatory variables together, was statistically significant, $p<0.001$. The strongest predictor of all types of falls except 'fall from the height' was female sex followed by the regular medicine use.

Conclusion: This study identified predictors for various types of falls in older people; the strongest predictor being a female sex followed by regular medicine use. Based on these findings, the medicine prescribing practice in this older population must be carefully reviewed. 


\section{INTRODUCTION}

Falls in older people (65 years of age and over) have been recognised as a major public health issue for more than past two decades (Hill \& Wee, 2012). Following a fall, the majority of older people are faced with lengthy periods of disability, requiring admission to geriatric rehabilitation services for a prolonged period of time (Hilmer et al., 2007). Past research has focused predominantly on psychotropic medicines as an intrinsic or endogenous risk factor for falls (Bloch et al., 2011; Hill \& Wee, 2012; Hilmer et al., 2007; Landi et al., 2005; Payne, Abel, Simpson, \& Maxwell, 2013). Traditionally, risk factors for falls have been categorised as extrinsic or exogenous (uneven or slippery floors, unsafe foot wear, poor lighting and loose rugs), intrinsic (co-morbidities, sensory changes, high risk medicines, polypharmacy) and behavioural (Hill \& Wee, 2012). Psychotropic medicines were identified as the leading cause of falls and previous studies have found that the risk of falls in older patients taking benzodiazepines is $44 \%$ higher than in patients not taking any psychotropic medicines (Ballokova et al., 2014; Mangoni \& Jackson, 2004). Mood stabilising agents, such as lithium and antipsychotic medicines, have also been reported to contribute to falls in older people (Chatterjee, Chen, Johnson, \& Aparasu, 2012; Ensrud et al., 2002). A recent study has found that cardiovascular medicines that affect blood pressure and the cardiac conducting system significantly increase the chance of falls in older people (Payne et al., 2013). One of the common adverse effects of psychotropic and antihypertensive agents is orthostatic hypotension. Thus, orthostatic hypotension may be an important risk factor for falls (Heitterachi, Lord, Meyerkort, McCloskey, \& Fitzpatrick, 2002); however, previous studies failed to demonstrate a correlation between falls and this adverse effect. 
Our study takes a different approach by examining the link between exogenous (medicines/polypharmacy and health status) and endogenous (age-dependent physiological changes and sex) factors that can be associated with falls in older people. Endogenous factors, including age-dependent decline in hepatic, renal and sensory function, appear to be more challenging to predict than exogenous factors, thus requiring more focus and attention to detail. In accordance with our model of assessing falls, it has been shown that effectiveness of the hepatic and renal system may play a crucial role in determining the incidence of falls. For example, medicines that are more easily metabolised by the liver and excreted through the renal system do not maintain concentration of drug metabolites in circulation for a long period of time (Rasmussen, Steentoft, Rasmussen, Kristensen, \& Moller, 1999). These metabolites that remain in the circulatory system can have adverse effects on the patient particularly in the case of psychotropic medicines (Ballokova et al., 2014). However, with an age-dependent decline in hepatic and renal function, the body's ability to remove drug metabolites is diminished, thus these metabolites can prolong the adverse effects of certain medicines and in turn lead to falls.

Long acting benzodiazepines as well as lithium can remain in circulation for long periods of time between doses (Berdot et al., 2009). When the ability to metabolise and eliminate these drugs decreases, their effects are prolonged causing dizziness, vertigo and other associated catalysts to falls. While this effect is most commonly associated with psychotropic medicines it is also prevalent in patients taking cardiovascular medicines. Therefore, parameters such glomerular filtration rate and liver enzyme values could provide valuable insight into the link between drug metabolism, elimination and falls (Berdot et al., 2009). Age-dependent physiological changes affecting renal and hepatic function have not previously been investigated in association with falls despite the evidence showing that a decline in drug metabolism leads to 
retention of drug metabolites in the circulation (Sproule, Naranjo, Brenmer, \& Hassan, 1997). Furthermore, the past studies focusing on the type of falls and predictors are limited (Gassmann, Rupprecht, \& Freiberger, 2009; Morris, 2007) and no links were made to whether a fall occurred on the flat/same level surface, height or steps or stairs. To the best of our knowledge, there are no studies that have investigated different types of falls in the context of age-dependent physiological changes.

The aim of this study is therefore to make a broad-spectrum analysis of older patients with falls by encompassing several possible factors such as health status, sex, polypharmacy and age-related changes in order to establish links between type of falls in older people and medicine use. Previous studies have attributed falls most commonly to the use of psychotropic medicines and no clinical audits were conducted to assess the dynamic relationship between age related factors, combination drug therapies and the type of falls that patients had. This study of older patients with falls takes into account the background factors related to their age such as glomerular filtration rate, liver function tests, and sensory function including any visual, vestibular or auditory changes and compare these factors to the use of different classes of medicines.

\section{METHODS}

\section{Study population and design}

Using a retrospective observational study design, the medical records of older patients admitted to a large scale academic hospital in Melbourne, Australia following a fall were examined. The study population included a random sample of 250 patients which comprised a $10 \%$ of the total number of patients $(n=2,492)$ admitted to the hospital following a fall between 
January 2013 and August 2014. The study was approved by the Human Research Ethics Committee of Melbourne Health, Australia (QA Number: QA2014053). Comprehensive data regarding patients' medicine history, health status and types of falls were collected from medical records. Data were collected using a hard copy of the data collection tool based on the following inclusion criteria: the patient was 65 years of age and over and the patient has experienced a fall related to hospital admission. The following details were documented and grouped as explanatory variables: age, sex, health conditions, and medicine history at the time of hospital admission which included all regular medicines taken by patients to manage their chronic condition, liver and renal function, and sensory function. These explanatory variables were then compared to the outcome variable which is the type of fall (unspecified/mechanical fall on the same level, fall from steps/stairs or height).

\section{Record of data}

Liver function was evaluated by reviewing the first set of test results during an older person's admission for three enzymes. Gamma-glutamyl transferase (GGT), aspartate transaminase (AST) and alanine transaminase (ALT) are commonly used as markers of liver dysfunction (Kunutsor, Apekey, \& Khan, 2014). Renal function was recorded as patients' estimated glomerular filtration rate (eGFR), which reflects the quantity of glomerular filtrate produced each minute by all nephrons in both kidneys (Zaman, Filipowicz, \& Beddhu, 2013). Sensory function data were obtained from falls risk assessment (FRA) records which indicated the ability of a patient to use sensory information to successfully perform day to day tasks such as vision, hearing and balance. Based on the exclusion criteria, which were patients with incomplete medicine history and patients with falls that resulted in fatality, data from 12 patients were excluded from the study, thus the total number of patients included in the study was 238 . 


\section{Statistical analysis}

Hard copy data were transferred and stored in a Microsoft Excel spreadsheet. Statistical analysis was performed using the IBM Statistical Package for the Social Sciences (SPSS, Version 22, Chicago, IL, USA). Descriptive statistical analyses were conducted on patients' age, sex, health status, types of falls, number and type of regular medicines used, sensory function, renal and hepatic function. Associations between explanatory variables and the outcome variable (type of fall) were investigated by running univariate analyses (Chi-square analysis) between each explanatory variable and the type of fall. Most explanatory variables were categorical in nature which related to their presence or absence (i.e. regular medicine use, prescription of analgesic, antihypertensive, psychotropic medication) whereas the following were continuous variables (i.e. age, eGFR, liver function tests - GGT, AST, ALT). This process was followed by performing multinominal logistic regression analysis between the variables showing associations at $\mathrm{p}<0.1$ in univariate analysis and the outcome variable (type of fall). In multinominal logistic regression analysis, a $\mathrm{p} \leq 0.05$ was used as a statistically significant value to identify independent associations between specific explanatory variables and the type of fall. Demographic data are presented as mean and standard deviation $(\mathrm{SD})$ results.

\section{RESULTS}

\section{Demographic characteristics: age, sex and health status}

The mean age of patients was 81.06 years $(\mathrm{SD}=8.3$ years) of age. The youngest patient was 65 years of age (the threshold for the study) while the oldest patient was 104 years old. Of the 238 patients, $104(44 \%)$ were male while $134(56 \%)$ were female patients. The health conditions patients suffered from were grouped into cardiovascular, hepatic, musculoskeletal, 
psychiatric, renal and other disorders. Of the 238 patients, $112(47 \%)$ did not have any preexisting psychiatric or cognitive disorder, while the remaining 126 (53\%) suffered from depression, anxiety, dementia and delirium. The most common psychiatric disorder was depression, affecting the largest proportion of the population with every 1 in 4 patients being affected. Additionally, of the 238 patients, 46 (19\%) did not have any cardiovascular, hepatic or renal disorder. The remaining 192 patients suffered from a range of conditions including hypertension (77\%), dyslipidaemia (41\%), atrial fibrillation and ischemic heart disease (20\%), and renal impairment (21\%). There were 129 patients who suffered from one or more preexisting musculoskeletal disorders while the remaining 109 patients did not have any musculoskeletal comorbidity. Of the 129 patients with musculoskeletal disorders, osteoarthritis and osteoporosis were the most common with $46 \%$ and $36 \%$ incidence respectively. Other disorders included type 2 diabetes mellitus (26\%) and thyroid gland abnormalities, such as hypothyroidism and hyperthyroidism $(6 \%)$.

\section{Medicine use}

Of the 238 patients, $229(96 \%)$ were on regular medicines. The mean number of medicines used was 6.08 ( $\mathrm{SD}=2.78$ medicines), with a minimum number used by a patient being one (1) and a maximum being 16. There were 8 classes of medicines commonly prescribed to 22 or more patients and these included analgesics, anti-platelets, anti-hypertensives, medicines affecting bone density, lipid lowering and oral hypoglycaemic drugs, proton pump inhibitors and psychotropics (Table 1). Anti-hypertensives were the most commonly prescribed with 162 (68\%) patients using multiple classes of anti-hypertensive medicines. Angiotensin $\left(\mathrm{AT}_{1}\right)$ receptor blockers such as irbesartan, candesartan and telmisartan were the most commonly used anti- 
hypertensives, followed by beta blockers (atenolol and metoprolol), and calcium channel blockers such as amlodipine and diltiazem (Table 1). Angiotensin converting enzyme (ACE) inhibitors and loop diuretics were prescribed to 45 and 36 patients, respectively. Paracetamol was the most commonly used analgesic to manage pain, followed by statins such as atorvastatin and simvastatin for dyslipidaemia. Medicines affecting bone such as alendronate and colecalciferol were prescribed to 74 patients whereas 89 patients were on psychotropic medicines with antidepressant use noted in 60 patients (67\%), followed by anxiolytics and hypnotics such as benzodiazepines (Table 1).

\section{Types of falls and associations with age and sex}

Using comprehensive clinical notes, the falls were categorized into five (5) types which were labelled from 1 to 5 as follows: 1-unspecified fall on the same level, 2-mechanical fall on the same level relating to slipping, tripping or loosing balance, 3-other unspecified fall on/from unspecified level such as fall from a chair, vehicle and fall as a result of syncope, 4-fall from steps or stairs, and 5-fall from the height (ladder, roof). The most common type of fall was the mechanical fall (35\%), followed by the unspecified fall on the same level (31\%), other type of fall (19\%), fall from steps or stairs (9\%) and fall from the height (6\%) (Table 2).

There was a significant association between the type of fall and sex, $\chi^{2}(4)=13.3$, $\mathrm{p}=0.01$, (Table 3) with females having more falls than males in each category except category 5 ; fall from the height (Table 2). Furthermore, there was no significant association between the type of fall and age, $\chi^{2}(4)=8.1, p=0.089$. The highest number of falls occurred in the $65-85$ age group (Table 2) and the most common type of fall was category 2 falls (mechanical fall on the same level relating to slipping, tripping or loosing balance). 
Associations between the type of fall, age-dependent physiological changes and medicines

Age-dependent physiological changes affecting patients in this study were placed under three (3) categories: sensory, renal and hepatic changes (Table 2). Sensory information data revealed that $59 \%$ of patients had normal sensory function whereas $41 \%$ of patients showed some form of sensory impairment with no significant association between the type of fall and sensory impairment. Furthermore, there was no significant association between the type of fall and eGFR with a minimum eGFR being 8 and maximum 95. Finally, there was no significant association between the type of fall and hepatic function as measured by GGT, ALT and AST (Table 3).

Chi-square analysis showed no association between the type of fall and medical conditions patients suffered from. In contrast there was a significant association between the type of fall and regular medicine use, $\chi^{2}(4)=16.7, p=0.002$ (Table 3). Additionally, there were significant associations between the type of fall and psychotropic medicines, $\chi^{2}(4)=10.8$, $\mathrm{p}=0.029$ and the medicines affecting the bone and type of fall, $\chi^{2}(4)=10.5, \mathrm{p}=0.033$ (Table 3 ). In regards to bone medicine use, 73 patients or $31 \%$ of the cohort was regularly taking one bone medicine and these patients had less falls in each category when compared to patients who were not taking bone medicines. Out of 238 patients, 89 (37\%) were taking psychotropic medicines, with a minimum being one and maximum being 4 . Only one patient was taking 4 whereas $61 / 89$ (69\%) were on one, 20/89 (23\%) were on two and 7/89 (8\%) were on three psychotropic medicines. 


\section{Multinominal logistic regression model of type of falls and explanatory variables}

The result of the multinominal logistic regression analysis showed that the full model which considered all the five explanatory (independent) variables together was statistically significant, $\chi^{2}(20)=55.8, p<0.001$. This result implied that the odds for a different type of fall were related to the five independent variables; sex, age, regular medicine use, bone and psychotropic medicines. The strongest predictor of all types of falls except 'fall from the height' was female sex with odds ratios (OR) being 5.3 for unspecified fall on the same level, 7.7 for mechanical fall on the same level relating to slipping, tripping or loosing balance, 7.6 unspecified fall on/from unspecified level such as fall from a chair, vehicle or syncope, and 9.0 for fall from steps or stairs (Table 4). The second predictor that made significant contribution to the model was the regular medicine use. Regular medicine use predicted an increased incidence of falls, $\mathrm{p}<0.001$. Thus, older individuals taking regular medicines were at increased risk of experiencing any type of fall identified in this study.

\section{DISCUSSION}

This study found that $96 \%$ of patients were on regular medicines, and the most commonly used were anti-hypertensive, analgesic and psychotropic medicines. This finding is in line with previous studies showing that older people regularly take multiple medicines daily

(Kerse et al., 2008) as co-morbidities become more prevalent with aging, thus the number of medicines increases. Females had more falls than males in each category except category 5, which includes a fall from the height. This finding suggests that males were possibly more likely to engage in risky behaviours or activities that require use of ladders, and that their decision making process and thus judgement could be possibly affected by use of multiple medicines. As 
shown here, there was a significant association between regular medicine use and falls, specifically bone and psychotropic medicines. Patients taking the medicines affecting bone had fewer falls than patients not taking these medicines with unspecified fall on the same level being the most common type of fall. This finding is in line with literature reporting that vitamin D supplementation can prevent falls, particularly in the vitamin D deficient older individuals (Winzenberg, van der Mei, Mason, Nowson, \& Jones, 2012). Additionally, bone medicines can prevent osteoporotic changes in postmenopausal women that could contribute to falls (Sanders \& Geraci, 2013). However, more research needs to be done in relation to prescribing practices around bone medicine group. Future studies should include details regarding the dose, frequency, length of administration, ethnicity, sex and a family history of bone disease. This information can provide an insight into the possibility of using this medicine group prophylactically much earlier in life in patients exhibiting strong predictors for falls.

The link between long term psychotropic medicine use and falls in older people has been well documented, however, previous studies have not taken into account the role different classes of medicines and age-dependent physiological changes play in falls. This study found that psychotropic medicines were prescribed to 89 patients. Antidepressant use was the highest at $67 \%$ and this finding correlates with the high proportion of patients having a depression as their pre-existing psychiatric disorder. Some classes of antidepressants such as selective serotonin and serotonin-noradrenaline reuptake inhibitors have long been associated with extrapyramidal adverse effects which commonly led to postural hypotension and subsequent falls (van Strien, Koek, van Marum, \& Emmelot-Vonk, 2013). Furthermore, psychotropic use is associated with complex regimens that can have an impact on older patients' ability to self-manage their medicines in terms of dose, frequency, and other specific recommendations and precautions 
(Bauer et al., 2013). Our study results showed that the patients had 4.7 times increased odds of experiencing a fall on the same level and 5.9 times increased odds of experiencing a fall from steps and stairs if they were on psychotropic medication compared to those who were not taking any psychotropics. However, the confidence intervals for these results were large and therefore the results were not significant. Further research should involve recruitment of patients with and without psychotropic medications to test the patterns in relation to various types of falls.

The ageing process causes various health-related complications and whilst some of these complications result in pathological changes, others can influence the physiology without actually resulting in a named co-morbidity. Hepatic and renal function have been shown to deteriorate with aging (Menkes, 1989) however our study showed no impact of hepatic and renal function on the type of fall experienced. Future studies should consider genetic influences, ethnicity and race, architectural features of people's homes and residential facilities and more specific renal function tests in the context of regular medicine use and the types of falls experienced not just whether the fall has occurred.

Using eGFR as a sole marker for renal function in hospitalised patients may not be true reflection of patients' renal state because eGFR can show day-to-day variability post-admission (Kanaoka et al., 2012). Our study suggests that in addition to eGFR, assessment of renal function in older people should also include more specific tests such as creatinine clearance. Creatinine clearance is calculated by using the Cockcroft-Gault equation that incorporates specific parameters such age, weight and sex into the equation (Fernandez-Prado, Castillo-Rodriguez, Velez-Arribas, Gracia-Iguacel, \& Ortiz, 2016). As a result of ageing process, older people have diminished skeletal muscle mass which can affect creatinine levels; creatinine is a by-product of the skeletal muscle (Denison, Cooper, Sayer, \& Robinson, 2015; Nowson \& O'Connell, 2015). 
Therefore, including age, sex and weight when assessing the renal function may control variability between older people and provide true reflection of individuals' renal state.

In conclusion, our study strengthens the evidence that connects psychotropic medicine use and falls in older people and identifies the additional predictors of sex that are related to different types of falls in the study population. These predictors were both exogenous (regular medicine use reflecting health status) and endogenous (sex) in nature. We found an association between sex and regular medicine use and different types of falls. Based on these findings, it is possible to make a recommendation that the prescription of medicines be carefully reviewed for patients with strong predictors for fall types. This review should be based on calculation of creatinine clearance in relation to renal function and the dose adjustment where relevant. Taken together, the findings of this study suggest: a) an urgent need for randomised controlled trials in older people especially with regard to antihypertensive and psychotropic medicine use in individuals with altered hepatic and renal function; and b) a review of the medicine prescribing practices in older people from different ethnic groups. The key limitation of this study is that it was retrospective in design transition of patients through different points of care could affect the medicine use and management and these discrepancies in medicine use could have affected the incidence of falls. Therefore, future studies must include representative sample of older people from different ethnic groups who reside in different settings. 


\section{ACKNOWLEDGEMENTS}

The authors gratefully thank the Health Information Services Officers of the hospital who were involved in retrieving medical records to enable data collection for the study.

\section{CONFLICT OF INTEREST}

None. The authors declare that they have no conflict of interest.

\section{FUNDING}

This research did not receive any specific grant from funding agencies in the public, commercial, or not-for-profit sectors. 


\section{REFERENCES}

Ballokova, A., Peel, N. M., Fialova, D., Scott, I. A., Gray, L. C., \& Hubbard, R. E. (2014). Use of benzodiazepines and association with falls in older people admitted to hospital: a prospective cohort study. Drugs \& Aging, 31(4), 299-310. doi: 10.1007/s40266-0140159-3

Bauer, M., Glenn, T., Alda, M., Sagduyu, K., Marsh, W., Grof, P., . . Whybrow, P. C. (2013). Regularity in daily mood stabilizer dosage taken by patients with bipolar disorder. Pharmacopsychiatry, 46(5), 163-168. doi: 10.1055/s-0033-1343398

Berdot, S., Bertrand, M., Dartigues, J. F., Fourrier, A., Tavernier, B., Ritchie, K., \& Alperovitch, A. (2009). Inappropriate medication use and risk of falls--a prospective study in a large community-dwelling elderly cohort. BMC Geriatrics, 9, 30. doi: 10.1186/1471-2318-930

Bloch, F., Thibaud, M., Dugue, B., Breque, C., Rigaud, A. S., \& Kemoun, G. (2011). Psychotropic drugs and falls in the elderly people: updated literature review and metaanalysis. Journal of Aging and Health, 23(2), 329-346. doi: 10.1177/0898264310381277

Chatterjee, S., Chen, H., Johnson, M. L., \& Aparasu, R. R. (2012). Risk of falls and fractures in older adults using atypical antipsychotic agents: a propensity score-adjusted, retrospective cohort study. American Journal of Geriatric Pharmacotherapy, 10(2), 8394. doi: 10.1016/j.amjopharm.2011.10.006

Denison, H. J., Cooper, C., Sayer, A. A., \& Robinson, S. M. (2015). Prevention and optimal management of sarcopenia: a review of combined exercise and nutrition interventions to 
improve muscle outcomes in older people. Clinical Interventions in Aging, 10, 859-869. doi: 10.2147/CIA.S55842

Ensrud, K. E., Blackwell, T. L., Mangione, C. M., Bowman, P. J., Whooley, M. A., Bauer, D. C., . . Nevitt, M. C. (2002). Central nervous system-active medications and risk for falls in older women. Journal of the American Geriatrics Society, 50(10), 1629-1637.

Fernandez-Prado, R., Castillo-Rodriguez, E., Velez-Arribas, F. J., Gracia-Iguacel, C., \& Ortiz, A. (2016). Creatinine Clearance Is Not Equal to Glomerular Filtration Rate and CockcroftGault Equation Is Not Equal to CKD-EPI Collaboration Equation. American Journal of Medicine, 129(12), 1259-1263. doi: 10.1016/j.amjmed.2016.08.019

Gassmann, K. G., Rupprecht, R., \& Freiberger, E. (2009). Predictors for occasional and recurrent falls in community-dwelling older people. Zeitschrift fur Gerontologie Geriatrie, 42(1), 3-10. doi: 10.1007/s00391-008-0506-2

Heitterachi, E., Lord, S. R., Meyerkort, P., McCloskey, I., \& Fitzpatrick, R. (2002). Blood pressure changes on upright tilting predict falls in older people. Age and Ageing, 31(3), 181-186.

Hill, K. D., \& Wee, R. (2012). Psychotropic drug-induced falls in older people: a review of interventions aimed at reducing the problem. Drugs \& Aging, 29(1), 15-30. doi: $10.2165 / 11598420-000000000-00000$

Hilmer, S. N., Mager, D. E., Simonsick, E. M., Cao, Y., Ling, S. M., Windham, B. G., . . . Abernethy, D. R. (2007). A drug burden index to define the functional burden of medications in older people. Archives of Internal Medicine, 167(8), 781-787. doi: 10.1001/archinte.167.8.781 
Kanaoka, T., Tamura, K., Ohsawa, M., Yanagi, M., Haku, S., Wakui, H., . . . Umemura, S. (2012). Relationship of ambulatory blood pressure and the heart rate profile with renal function parameters in hypertensive patients with chronic kidney disease. Clinical and Experimental Hypertension, 34(4), 264-269. doi: 10.3109/10641963.2012.681082

Kerse, N., Flicker, L., Pfaff, J. J., Draper, B., Lautenschlager, N. T., Sim, M., . . Almeida, O. P. (2008). Falls, depression and antidepressants in later life: a large primary care appraisal. PLoS One, 3(6), e2423. doi: 10.1371/journal.pone.0002423

Kunutsor, S. K., Apekey, T. A., \& Khan, H. (2014). Liver enzymes and risk of cardiovascular disease in the general population: a meta-analysis of prospective cohort studies. Atherosclerosis, 236(1), 7-17. doi: 10.1016/j.atherosclerosis.2014.06.006

Landi, F., Onder, G., Cesari, M., Barillaro, C., Russo, A., \& Bernabei, R. (2005). Psychotropic medications and risk for falls among community-dwelling frail older people: an observational study. Journals of Gerontology Series A, Biological Sciences and Medical Sciences, 60(5), 622-626.

Mangoni, A. A., \& Jackson, S. H. (2004). Age-related changes in pharmacokinetics and pharmacodynamics: basic principles and practical applications. British Journal of Clinical Pharmacology, 57(1), 6-14.

Menkes, C. J. (1989). Renal and hepatic effects of NSAIDs in the elderly. Scandinavin Journal of Rheumatology Supplement, 83, 11-13.

Morris, R. (2007). Predicting falls in older women. Menopause International, 13(4), 170-177. doi: $10.1258 / 175404507783004131$

Nowson, C., \& O'Connell, S. (2015). Protein Requirements and Recommendations for Older People: A Review. Nutrients, 7(8), 6874-6899. doi: 10.3390/nu7085311 
Payne, R. A., Abel, G. A., Simpson, C. R., \& Maxwell, S. R. (2013). Association between prescribing of cardiovascular and psychotropic medications and hospital admission for falls or fractures. Drugs \& Aging, 30(4), 247-254. doi: 10.1007/s40266-013-0058-z

Rasmussen, L. S., Steentoft, A., Rasmussen, H., Kristensen, P. A., \& Moller, J. T. (1999). Benzodiazepines and postoperative cognitive dysfunction in the elderly. ISPOCD Group. International Study of Postoperative Cognitive Dysfunction. British Journal of Anaesthesia, 83(4), 585-589.

Sanders, S., \& Geraci, S. A. (2013). Osteoporosis in postmenopausal women: considerations in prevention and treatment: (women's health series). Southern Medical Journal, 106(12), 698-706. doi: 10.1097/SMJ.0b013e3182a0df8b

Sproule, B. A., Naranjo, C. A., Brenmer, K. E., \& Hassan, P. C. (1997). Selective serotonin reuptake inhibitors and CNS drug interactions. A critical review of the evidence. Clinical Pharmacokinetics, 33(6), 454-471.

van Strien, A. M., Koek, H. L., van Marum, R. J., \& Emmelot-Vonk, M. H. (2013). Psychotropic medications, including short acting benzodiazepines, strongly increase the frequency of falls in elderly. Maturitas, 74(4), 357-362. doi: 10.1016/j.maturitas.2013.01.004

Winzenberg, T., van der Mei, I., Mason, R. S., Nowson, C., \& Jones, G. (2012). Vitamin D and the musculoskeletal health of older adults. Australian Family Physician, 41(3), 92-99.

Zaman, T., Filipowicz, R., \& Beddhu, S. (2013). Implications and importance of skeletal muscle mass in estimating glomerular filtration rate at dialysis initiation. Journal of Renal Nutrition, 23(3), 233-236. doi: 10.1053/j.jrn.2013.01.028 
Table 1 Distribution of regular medicine prescription according to the clinical composition of the sample.

\begin{tabular}{|c|c|c|}
\hline Clinical indication & Medication class & Number of prescriptions \\
\hline Coronary syndrome & Anti-platelets (Aspirin) & 90 \\
\hline Dyslipidaemia & Statins & 110 \\
\hline \multirow[t]{5}{*}{ Hypertension } & $\begin{array}{l}\text { Angiotensin }\left(\mathrm{AT}_{1}\right) \text { receptor } \\
\text { blockers }\end{array}$ & 72 \\
\hline & Beta blockers & 51 \\
\hline & Calcium-channel blockers & 48 \\
\hline & ACE inhibitors & 45 \\
\hline & Loop diuretics & 36 \\
\hline \multirow[t]{2}{*}{ Osteoporosis } & Vitamin D & 60 \\
\hline & Bisphosphonates & 14 \\
\hline \multirow[t]{2}{*}{ Pain } & Analgesics (Paracetamol) & 133 \\
\hline & Opiod analgesics & 59 \\
\hline \multirow[t]{5}{*}{ Psychiatric disorders } & Benzodiazepines & 28 \\
\hline & $\begin{array}{l}\text { Selective serotonin } \\
\text { reuptake inhibitors }\end{array}$ & 24 \\
\hline & Tetracyclic antidepressants & 15 \\
\hline & Serotonin-noradrenaline & \\
\hline & reuptake inhibitors & 11 \\
\hline Reflux disease & Proton pump inhibitors & 91 \\
\hline \multirow[t]{2}{*}{ Type 2 diabetes } & Biguanides & 27 \\
\hline & Sulfonylureas & 19 \\
\hline
\end{tabular}


Table 2 Distribution of explanatory variables according to the type of fall.

\begin{tabular}{|c|c|c|c|c|c|}
\hline $\begin{array}{l}\text { Sample } \\
\text { characteristics } \\
(\mathrm{N}=238)\end{array}$ & $\begin{array}{l}\text { Unspecified } \\
\text { fall on the } \\
\text { same level }(n)\end{array}$ & $\begin{array}{l}\text { Mechanical } \\
\text { fall on the } \\
\text { same level } \\
(n)\end{array}$ & $\begin{array}{l}\text { Fall from } \\
\text { unspecified } \\
\text { level }(n)\end{array}$ & $\begin{array}{l}\text { Fall from } \\
\text { steps or } \\
\text { stairs }(n)\end{array}$ & $\begin{array}{l}\text { Fall from the } \\
\text { height }(n)\end{array}$ \\
\hline \multicolumn{6}{|l|}{ Gender } \\
\hline Male & 34 & 32 & 17 & 8 & 13 \\
\hline Female & 40 & 51 & 29 & 12 & 2 \\
\hline \multicolumn{6}{|l|}{ Age group } \\
\hline $65-85$ y & 48 & 53 & 30 & 14 & 15 \\
\hline $86-104$ y & 26 & 30 & 16 & 6 & 0 \\
\hline \multicolumn{6}{|c|}{ Regular medicine use } \\
\hline No & 1 & 2 & 2 & 4 & 0 \\
\hline Yes & 73 & 81 & 44 & 16 & 15 \\
\hline \multicolumn{6}{|c|}{ Anti-hypertensives } \\
\hline No & 24 & 27 & 10 & 8 & 7 \\
\hline Yes & 50 & 56 & 36 & 12 & 8 \\
\hline \multicolumn{6}{|l|}{ Lipid-lowering } \\
\hline No & 41 & 41 & 27 & 12 & 10 \\
\hline Yes & 33 & 42 & 19 & 8 & 5 \\
\hline \multicolumn{6}{|l|}{ GI medicines } \\
\hline No & 49 & 54 & 30 & 13 & 9 \\
\hline Yes & 25 & 29 & 16 & 7 & 6 \\
\hline \multicolumn{6}{|l|}{ Psychotropics } \\
\hline No & 38 & 59 & 28 & 11 & 13 \\
\hline Yes & 36 & 24 & 18 & 9 & 2 \\
\hline \multicolumn{6}{|l|}{ Bone medicines } \\
\hline No & 54 & 53 & 27 & 17 & 14 \\
\hline Yes & 20 & 30 & 19 & 3 & 1 \\
\hline \multicolumn{6}{|l|}{ Sensory function } \\
\hline Normal & 39 & 45 & 19 & 10 & 10 \\
\hline \multicolumn{6}{|l|}{ eGFR } \\
\hline Normal & 10 & 2 & 4 & 0 & 1 \\
\hline Impaired & 34 & 34 & 20 & 6 & 3 \\
\hline \multicolumn{6}{|l|}{ GGT } \\
\hline Normal & 20 & 16 & 10 & 2 & 2 \\
\hline Impaired & 12 & 12 & 8 & 1 & 1 \\
\hline \multicolumn{6}{|l|}{ ALT } \\
\hline Normal & 29 & 27 & 14 & 2 & 2 \\
\hline Impaired & 9 & 3 & 6 & 1 & 1 \\
\hline \multicolumn{6}{|l|}{ AST } \\
\hline Normal & 25 & 26 & 15 & 1 & 2 \\
\hline Impaired & 11 & 4 & 5 & 2 & 1 \\
\hline
\end{tabular}

$N=$ total number of subjects in the study

$n=$ number of subjects experiencing specific type of fall 
Table 3 Associations between the type of fall, age, gender, age-dependent physiological changes and regular medicine use.

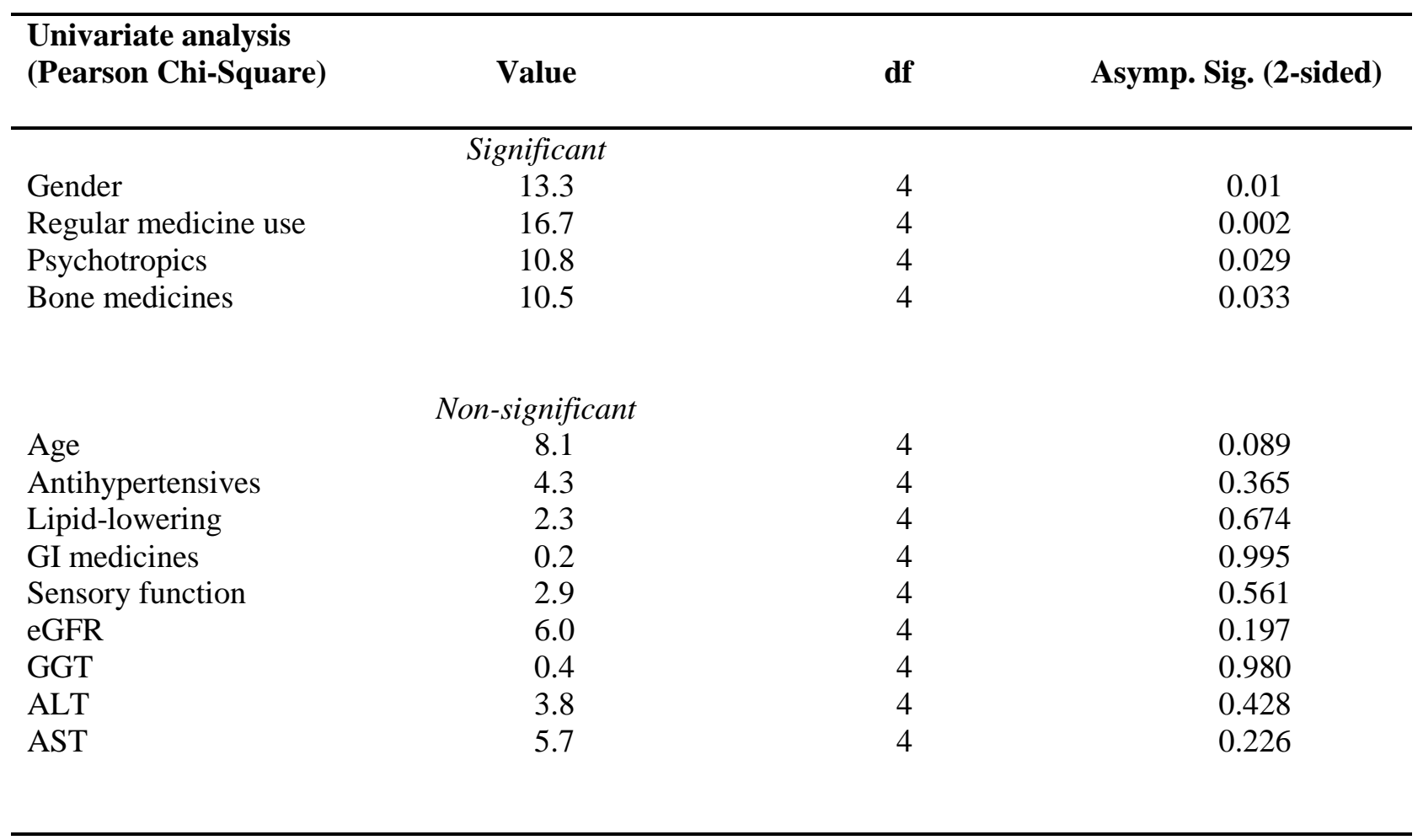


Table 4 Multinominal logistic regression predicting the likelihood of different types of falls.

\begin{tabular}{lccc}
\hline Type of fall & P value & OR & 95\% CI \\
& & & \\
& & & \\
Unspecified fall on the same level & 0.04 & 5.3 & $1.1-26.4$ \\
Female & $<0.001$ & $2.1 \times 10^{7}$ & $1.9 \times 10^{8}-2.3 \times 10^{6}$ \\
Regular medicine use & 0.06 & 4.7 & $0.9-23.2$ \\
Psychotropics & & & \\
Mechanical fall on the same level & 0.012 & 7.7 & $1.6-37.6$ \\
Female & $<0.001$ & $1.3 \times 10^{7}$ & $1.9 \times 10^{8}-8.9 \times 10^{7}$ \\
Regular medicine use & & & \\
& 0.016 & 7.6 & $1.5-39.7$ \\
Fall from unspecified level & $<0.001$ & $5.5 \times 10^{8}$ & $7.9 \times 10^{9}-3.8 \times 10^{7}$ \\
Female & & & \\
Regular medicine use & & & \\
& 0.018 & 9.0 & $1.5-55.4$ \\
Fall from steps or stairs & 0.06 & 5.9 & $0.9-36.8$ \\
Female & & & \\
Psychotropics & & & \\
\hline
\end{tabular}

${ }^{\text {Note }}$ Fall from a height was taken as a reference point for regression analysis. 